\title{
Evaluation of $\alpha_{2}$-Integrin Expression as a Biomarker for Tumor Growth Inhibition for the Investigational Integrin Inhibitor E7820 in Preclinical and Clinical Studies
}

\author{
Ron J. Keizer, ${ }^{1,6}$ Y. Funahashi, ${ }^{2}$ T. Semba, ${ }^{3}$ J. Wanders, ${ }^{4}$ J. H. Beijnen, ${ }^{1,5}$ \\ J. H. M. Schellens, ${ }^{4,5}$ and A. D. R. Huitema ${ }^{1}$
}

\begin{abstract}
Received 17 August 2010; accepted 4 February 2011; published online 9 March 2011
Abstract. E7820 is an orally active inhibitor of $\alpha_{2}$-integrin mRNA expression, currently tested in phases I and II. We aimed to evaluate what levels of inhibition of integrin expression are needed to achieve tumor stasis in mice, and to compare this to the level of inhibition achieved in humans. Tumor growth inhibition was measured in mice bearing a pancreatic KP-1 tumor, dosed at $12.5-200 \mathrm{mg} / \mathrm{kg}$ over 21 days. In the phase I study, E7820 was administered daily for 28 days over a range of 0-200 $\mathrm{mg}$, followed by a 7-day washout period. PK-PD models were developed in NONMEM. $\alpha_{2}$-Integrin expression measured on platelets, corresponding to tumor stasis at $t=21$ in $50 \%$ and $90 \%$ of the mice $\left(I_{\text {int }, 50}, I_{\text {int, } 90}\right)$ were calculated. It was evaluated if these levels of inhibition could be achieved in patients at tolerable doses. One hundred nineteen $\alpha_{2}$-Integrin measurements and 210 tumor size measurements were available from mice. The relationship between PK and $\alpha_{2}$-integrin expression was modeled using an indirect-effect model, subsequently linked to an exponential tumor growth model. $I_{\text {inh,50 }}$ and $I_{\text {inh, } 90}$ were $14.7 \%$ (RSE $7 \%$ ) and $17.9 \%$ (RSE 8\%). Four hundred sixty two $\alpha_{2}$-integrin measurements were available from 29 patients. Using the schedule of $100 \mathrm{mg}$ qd (MTD), $\alpha_{2}$-integrin expression was inhibited more strongly than the $I_{\text {int, } 50}$ and $I_{\text {int }, 90}$ in greater than $95 \%$ and greater than $50 \%$ of patients, respectively. Moderate inhibition of $\alpha_{2}$-integrin expression corresponded to tumor stasis in mice, and similar levels could be reached in patients with the dose level of $100 \mathrm{mg}$ qd.
\end{abstract}

KEY WORDS: biomarker; E7820; modeling and simulation; oncology; pharmacodynamics.

\section{INTRODUCTION}

The investigational anti-cancer drug E7820 is an orally active angiogenesis inhibitor, and has shown anti-tumor activity in several tumor models in mice, the effect of which was mediated through the inhibition of expression of $\alpha_{2^{-}}$ integrin $(1,2)$. Integrins are receptors that mediate attachment between cells, and between cells and the extracellular matrix (3). They also play an important role in cell signaling. Many types of integrins have been identified, multiple types may be expressed on the cell surface simultaneously. Integrins are

\footnotetext{
${ }^{1}$ Department of Pharmacy and Pharmacology, The Netherlands Cancer Institute/Slotervaart Hospital, Louwesweg 6, 1066 EC, Amsterdam, The Netherlands.

${ }^{2}$ Research Laboratories Eisai Co., Ltd., Ibaraki, Japan.

${ }^{3}$ Eisai Co., Ltd, Hatfield, Hertfordshire, UK.

${ }^{4}$ Division of Clinical Pharmacology, Department of Medical Oncology, The Netherlands Cancer Institute, Amsterdam, The Netherlands.

${ }^{5}$ Division of Drug Toxicology, Section of Biomedical Analysis, Department of Pharmaceutical Sciences, Faculty of Science, Utrecht University, Utrecht, The Netherlands.

${ }^{6}$ To whom correspondence should be addressed. (e-mail: ron. keizer@slz.nl)
}

heterodimers, consisting of $\alpha$ (alpha) and $\beta$ (beta) subunits. In mammals, $18 \alpha$ and $8 \beta$ subunits have been characterized, with varying functions related to cell attachment and cell signaling. It has been shown in preclinical experiments that suppression of integrin $\alpha_{2}$ by E7820 played a crucial role in inhibition of endothelium tube formation $(1,2)$. E7820 was evaluated in a phase I dose escalation study in patients with malignant solid tumors or lymphomas, the clinical results of which have been reported previously (4) and for which a population PK model was presented before (5). Since $\alpha_{2}$ integrin is expressed both on tumor cells and on platelets, it has been hypothesized that $\alpha_{2}$-integrin expression on platelets may be an easily evaluable biomarker for tumor growth inhibition in response to treatment with E7820 $(1,2)$.

The inhibition of $\alpha_{2}$-integrin measured on platelets provides a measure of pharmacological target modulation, and therefore would theoretically provide a better predictor of activity than measures of E7820 plasma exposure. $\alpha_{2}$ Integrin is therefore currently being evaluated in phase I studies as possible biomarker $(4,6)$. In this article, we present a modeling and simulation analysis that integrates data from preclinical experiments and a phase I clinical trial to evaluate the expected efficacy of the dose levels that were studies in phase I. Similar analyses have been presented earlier for the development of everolimus (7) and gefitinib (8). The aim of 
the analysis was to establish levels of integrin inhibition in humans which corresponded to inhibition levels associated with tumor stasis in mice. Therefore, we first aimed to establish what level of $\alpha_{2}$-integrin inhibition corresponds to tumor stasis in mice. This was done by establishing PK-PD models for the preclinical experiments, describing the relationships between E7820 plasma exposure, the inhibition of $\alpha_{2}$-integrin expression, and tumor growth inhibition. Subsequently, we investigated if the dose regimen proposed from the phase I study based on acceptable toxicity would result in sufficient inhibition of $\alpha_{2}$-integrin expression to expect antitumor activity in patients. For this, a model was constructed describing effects of exposure to E7820 on $\alpha_{2}$-integrin expression. This model was based on the human PK model and measurements of $\alpha_{2}$-integrin during the phase I trial. Finally, it was evaluated which clinical regimens were capable of achieving inhibition of $\alpha_{2}$ integrin expression in humans similar to that which led to tumor stasis in mice. It was thus assumed that a similar level of $\alpha_{2}$-integrin expression would be required for tumor growth inhibition in mice and humans, for tumors that are sensitive to angiogenesis inhibition mediated by $\alpha_{2}$-integrin.

\section{METHODS}

\section{Preclinical Experiments}

The PK profile of E7820 in female KSN Slc mice aged 6, 7 , or 8 weeks was elucidated after single IV (25 mg/kg, $n=3)$, single oral $(25,50$, and $100 \mathrm{mg} / \mathrm{kg}, n=4)$, and seven repeated oral administrations with about $12 \mathrm{~h}$ intervals $(50 \mathrm{mg} / \mathrm{kg}, n=$ 4). Concentrations of E7820 in plasma were obtained using a validated liquid chromatography method with UV detection, with precision $<15 \%$ over the entire concentration range. Mean PK parameters $C L, V$, and $F$ were calculated by noncompartmental analysis. The tumor growth experiments in mice have been described before (1). Briefly, a human pancreatic carcinoma cell line $\left(\mathrm{KP}-1,5 \times 10^{6}\right.$ cells/head) was transplanted subcutaneously into 7 -week old female nude mice. Administration of E7820 was started at doses of 12.5, $25,50,100$, or $200 \mathrm{mg} / \mathrm{kg}$, or vehicle, 1 week after the transplantation. E7820 was administered orally by gavage, twice a day for 3 weeks. PK samples were collected twice weekly. Blood was withdrawn once a week from the eye of anesthesized mice in PBS containing $0.004 \%$ sodium citrate and diluted at 1:100. Diluted blood samples were directly stained with FITC-conjugated anti-integrin $\alpha 2 \mathrm{Ab}$ and expression levels on platelets were analyzed by flow cytometry. The longest diameter of the tumor and body weight was measured twice a week by direct measurement of the tumor diameters with calipers.

\section{Software}

The statistical data analysis and simulations were performed with non-linear mixed-effects modeling using NONMEM, version VI, level 2.0 (Icon Development Solutions, Ellicott City, MD, USA) with gfortran (http://gcc.gnu.org/ fortran/) as Fortran compiler, and Piraña (9) as modeling environment. The first-order conditional estimation method with interaction (FOCE-I) was used throughout the model building. Standard errors for model parameters were estimated using the covariance step in NONMEM. Model evaluation and selection was based on objective function value ( $p$ level of $0.01, \Delta \mathrm{OFV}=6.63$ was considered a significant improvement in fit), successful convergence, goodness-of-fit plots and visual predictive checks (10). R (version 2.9.0, http://www.cran.r-project.org) was used for the analysis of simulated data, and the generation of plots. Modeling and simulation were performed according to a pre-specified analysis plan, and construction of the PK-PD model was performed sequentially. First, the effect of E7820 plasma concentrations was correlated with the inhibition of $\alpha_{2}$ integrin expression. Next, a tumor growth model based on the unperturbed tumor growth experiment was fitted. The parameter estimates for the $\alpha_{2}$-integrin model were then fixed, and the model predicted expression levels were used to drive the different tumor growth models that were evaluated.

\section{Integrin Expression in Mice}

Longitudinal description of the $\alpha_{2}$-integrin expression level on platelets was modeled as an indirect response model (11) with an inhibitory effect on input rate $\left(k_{\text {in }}\right)$ of E7820 plasma concentration, described by a linear equation or by the Hill equation:

$$
\frac{d I}{d t}=k_{\mathrm{in}} \cdot\left(1-\frac{E_{\mathrm{max}, I} \cdot c_{p}^{\gamma}}{\mathrm{IC}_{50, I}^{\gamma}+c_{p}^{\gamma}}\right)-k_{\mathrm{out}} \cdot I
$$

in which $I$ describes the relative integrin expression, $k_{\text {in }}$ and $k_{\text {out }}$ are the input and output rates of the indirect-effect model. The plasma concentration of E7820 was defined as $c_{\mathrm{p}}$, and $E_{\text {max,I }}, \mathrm{IC}_{50}$ and $\gamma$ were the maximal drug effect, the plasma concentration at half maximal effect, and the shape exponent of the Hill equation describing the drug effect on the $\alpha_{2}$-integrin expression rate, respectively. An inhibitory effect of plasma exposure on $k_{\text {in }}$ was considered a mechanistically more plausible model than a stimulatory effect on $k_{\text {out }}$ as the pharmacological effect of E7820 is mediated through the inhibition of mRNA expression. $\alpha_{2}$-Integrin expression at baseline $\left(I_{0}\right)$ was estimated (12). To allow for hysteresis in drug effects on $\alpha_{2}$-integrin, the inclusion of one or more effect compartments was tested. It was also assessed if development of tolerance to E7820 could be shown.

\section{Tumor Growth in Mice}

Two prerequisites for the tumor growth model were established: the model should be able to (a) capture the unperturbed growth, and (b) capture the inhibitory effect of decreased $\alpha_{2}$-integrin expression on tumor growth/shrinkage rate, over the entire dose range of E7820. Several tumor growth models were evaluated, including exponential models, Gompertz models (13), and a tumor growth model introduced by Simeone et al. (14). Linear and $E_{\max }$ relationships were evaluated for their ability to correlate the effect of inhibition of $\alpha_{2}$-integrin expression to one of the relevant growth rate parameters in the tumor growth models. For both the model for $\alpha_{2}$-integrin and the tumor growth model, it was evaluated if the available data supported the estimation of between 
subject variability (BSV) of the parameters. For both models, exponential residual error model were used.

\section{Calculation of Efficacious $\alpha_{2}$-Integrin Inhibition}

Using stochastic simulations from the preclinical PK-PD model, it was investigated what level of inhibition of expression of $\alpha_{2}$-integrin correlated with tumor stasis. Achieving tumor stasis at $t=21$ days (end of treatment period in preclinical experiments) in $50 \%$ or $90 \%$ of mice were defined as targets. The inhibition of $\alpha_{2}$-integrin expression $\left(I_{\text {int,av }}\right)$ at steady state was chosen as the measure of inhibition of $\alpha_{2}$-integrin expression required to achieve these goals. The simulations were performed for dose levels varying from 50 to $200 \mathrm{mg} / \mathrm{kg}$ with $20-\mathrm{mg} / \mathrm{kg}$ intervals, and each simulations was performed for 500 mice. In each simulation, the percentage of mice having tumor sizes less than or equal to their baseline tumor sizes was recorded, along with the $I_{\text {inh,av. From the }}$ results of the simulations, relationships between dose level, $\alpha_{2}$-integrin inhibition and tumor growth inhibition at $t=21$ were plotted, and the $I_{\text {int,av }}$ values at which $50 \%$ and $90 \%$ of mice achieved tumor stasis were calculated ( $I_{\text {inh,90 }}$ and $\left.I_{\text {inh,50 }}\right)$. To account for uncertainty in model parameter estimates, and to calculate relative standard errors (RSE) for these targets, these simulations were repeated 250 times with parameters sampled from the variance-covariance matrix obtained for the model.

\section{Clinical Trial Data}

E7820 was administered to patients daily for 28 days, followed by a washout period of 7 days prior to starting subsequent cycles. Blood samples were collected from 1 up to 9 treatment cycles. Bioanalysis of PK was performed using the same method as used for the preclinical samples. Blood samples for $\alpha_{2}$-integrin expression were determined from blood collected at predose, and at $6 \mathrm{~h}$ post-drug administration on day 1 , predose on day 28 of cycle 1 and at $24 \mathrm{~h}$ post-drug administration. Beyond cycle 1 and for each subsequent cycle, blood was collected on day 28 predose. Diluted blood samples were directly stained with FITCconjugated anti-integrin $\alpha 2 \mathrm{Ab}$ and expression levels on platelets were analyzed by flow cytometry, and expressed in molecules of equivalent soluble fluorochrome.

\section{Modeling of Clinical Data}

Previously, a population PK model was constructed from data from the phase I trial (Table I) (5). This model was used here as well, however, to avoid difficulties arising from incorporation of multiple dosing in the turnover absorption model, the absorption model was simplified to a first-order absorption model. The empirical Bayesian estimates (EBEs) for oral clearance $(\mathrm{CL} / F)$ and volume of distribution $(V / F)$, and typical parameter values for ka obtained from this PK model and the observed data were used to drive the PD model for $\alpha_{2}$-integrin expression. Within-subject variability in PK parameters was disregarded in the PD analysis. For the clinical data, a similar indirect-effect model for the inhibition of $\alpha_{2}$-integrin expression was evaluated as was used for the preclinical data. It was evaluated if the available data supported the estimation of BSV on the PD parameters. Dosing regimens of 50, 70, 100, and $200 \mathrm{mg}$ qd were then simulated using the developed model to investigate the expected inhibition of $\alpha_{2}$-integrin expression during continuous treatment at these levels. These simulations were performed in 1,000 patients, to account for inter individual variability in response to E7820. The relative $\alpha_{2}$-integrin inhibition at steady state was calculated (two cycles), which was compared to the $I_{\text {inh,av }}$ that was required for tumor stasis calculated from the preclinical data.

\section{RESULTS}

\section{PK in Mice}

In the PK experiments in mice, elimination half-life was $0.63 \mathrm{~h}$ and a low plasma clearance $\left(\mathrm{CL}=7.85 \mathrm{~mL} \mathrm{~min}{ }^{-1} \mathrm{~kg}^{-1}\right)$ and low volume of distribution $(V=0.684 \mathrm{~L} / \mathrm{kg})$ after IV dosing of E7820 were observed. The observed PK profiles after IV administration were monophasic, and a linear increase of AUC was observed from 25 to $100 \mathrm{mg} / \mathrm{kg}$ after a single oral dose. As the absorption was rapid with a $t_{\max }$ at $\sim 1 \mathrm{~h}$, an absorption rate (ka) of $3 \mathrm{~h}^{-1}$ was used throughout. Plasma protein binding of E7820 was concentration independent from 5 to $100 \mathrm{mg} / \mathrm{L}$, and was $89 \%$ (range 88.0 $90.2 \%)$. Mean bioavailability after oral administration of $25 \mathrm{mg} / \mathrm{kg}$ was $61.3 \%$. E7820 showed anti-tumor activity at doses of 50,100, and $200 \mathrm{mg} / \mathrm{kg}$ in the tumor growth and $\alpha_{2^{-}}$ integrin expression experiments.

\section{Integrin Inhibition in Mice}

The exposure-response relationship between E7820 plasma concentration and the inhibition of input rate of the turnover model was significantly better described using an $E_{\max }$-equation than when using a linear relationship. An $E_{\max }$ value of 1.1 (RSE 36\%) was estimated, but was not significantly different from 1 . Likely, due to the fact that $\alpha_{2^{-}}$ integrin expression was inhibited maximally by only $30 \%$, a sigmoidal $E_{\max }$ model could not be estimated. Therefore, the

Table I. Characteristics of Clinical Phase I Study (4)

\begin{tabular}{lll}
\hline & & Value (range) \\
\hline Total number of patients & 36 \\
Dose level & $10 \mathrm{mg}$ & 3 \\
& $20 \mathrm{mg}$ & 4 \\
& $40 \mathrm{mg}$ & 3 \\
& $70 \mathrm{mg}$ & 3 \\
& $100 \mathrm{mg}$ & 17 \\
Sex & $200 \mathrm{mg}$ & 6 \\
& Male & 20 \\
Race & Female & 16 \\
& Caucasian & 31 \\
Weight $(\mathrm{kg})$ & Black & 1 \\
Height $(\mathrm{cm})$ & Hispanic & 4 \\
BSA $\left(\mathrm{m}^{2}\right)$ & & $70.9(43.2-113.6)$ \\
Age $($ years $)$ & & $167.3(150.5-182.9)$ \\
\hline
\end{tabular}

Continuous variables given as mean (range) and categorical data as counts 
shape parameter $\gamma$ in the Hill equation was fixed to 1 , reducing the exposure-response relationship to a nonsigmoid $E_{\max }$ model. The inclusion of delay in the effects of drug exposure on $\alpha_{2}$-integrin expression did not significantly improve model fit, nor did the inclusion of a term describing resistance to the inhibition of $\alpha_{2}$-integrin expression by E7820. Figure 1 shows a visual predictive check of the model describing $\alpha_{2}$-integrin expression, which indicated adequate model fit.

\section{Tumor Growth in Mice}

Although all evaluated tumor growth models were able to describe unperturbed growth, only when an exponential model was used could the effects of integrin inhibition be captured adequately over the entire dose range studied in the preclinical experiments. In the exponential model, it was however necessary to include an adjustment for the observed initial slow tumor growth, since almost no tumor growth or even tumor shrinkage in some mice (receiving only vehicle) was observed in the first week. This was captured by including a mono-exponential term describing an initially complete and gradually decreasing resistance to tumor growth, on top of the basic exponential tumor growth differential equation:

$$
\frac{d T}{d t}=\alpha \cdot\left(1-e^{-\beta \cdot t}\right) \cdot T
$$

with $T$ describing the tumor size in $\mathrm{mm}, \alpha$ describing the tumor growth rate, $\beta$ being the rate of decline of initial resistance to maximal unperturbed tumor growth, and $t$ being the time in days after start of treatment. The inhibitory effect of decreased $\alpha_{2}$-integrin expression on tumor growth was incorporated as a sigmoidal $E_{\max }$ function, implemented relative to current tumor size:

$$
\frac{d T}{d t}=\alpha \cdot\left(1-e^{-\beta \cdot t}\right) \cdot T-\frac{E_{\mathrm{max}, T} \cdot I_{\mathrm{inh}, t}^{\gamma}}{\mathrm{II}_{50}^{\gamma}+I_{\mathrm{inh}, t}^{\gamma}} \cdot T
$$

$E_{\max , T}, \mathrm{II}_{50}$ and $\gamma$ are the maximal effect of inhibition of $\alpha_{2}$-integrin expression, the plasma concentration at half maximal effect, and the Hill coefficient, respectively. The Hill coefficient could not be estimated, and was therefore fixed at 5 to allow sigmoidicity in the relationship. This provided significantly better fit than using the non-sigmoidal form. The inclusion of a delay between the effect of $\alpha_{2}$-integrin inhibition on tumor growth did not result in a significantly better model fit. All parameters could be estimated with reasonable precision, except the parameters describing unperturbed growth. The high uncertainty $(>100 \%)$ in estimation of these parameters could be explained by the fact that these estimates were obtained by fitting only the tumor growth data from the five mice that received vehicle and no drug. A visual predictive check, shown in Fig. 2, however showed that the model described the observed data accurately for these mice, and also for the other mice, when the effects of decreased $\alpha_{2}$-integrin expression were incorporated in the model.

A schematic representation of the structural model describing the tumor growth inhibition and $\alpha_{2}$-integrin expression is shown in Fig. 3, and parameter estimates for the PK-PD model are presented in Tables II, III, and IV. The tumor growth model was able to describe the tumor growth in

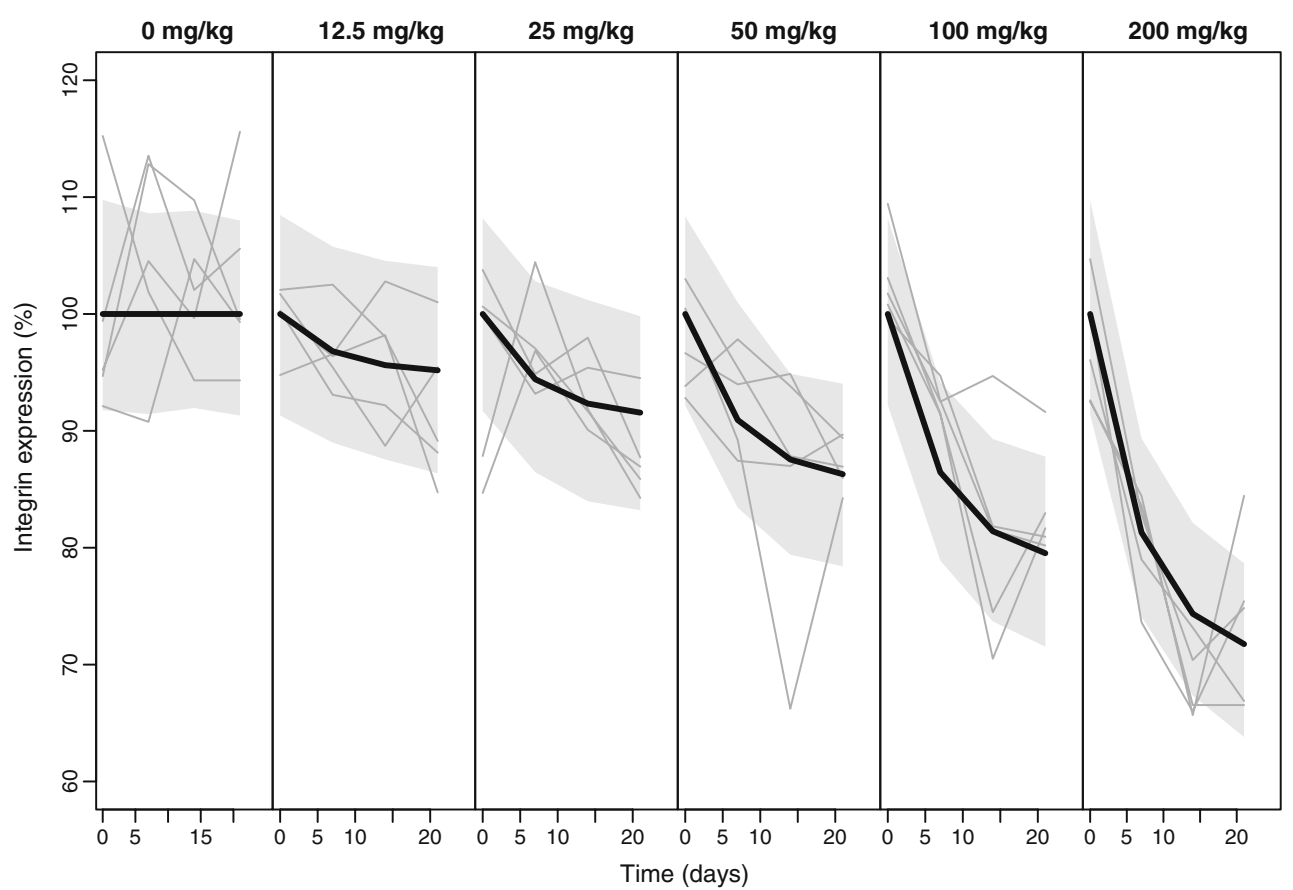

Fig. 1. $\alpha_{2}$-Integrin expression profiles in mice with implanted KP-1 tumors at different dose levels of E7820. The thin solid lines indicate observed growth curves, the black solid line indicates the model predicted growth curve, and the gray areas indicate the $80 \%$ confidence interval of the model predictions 


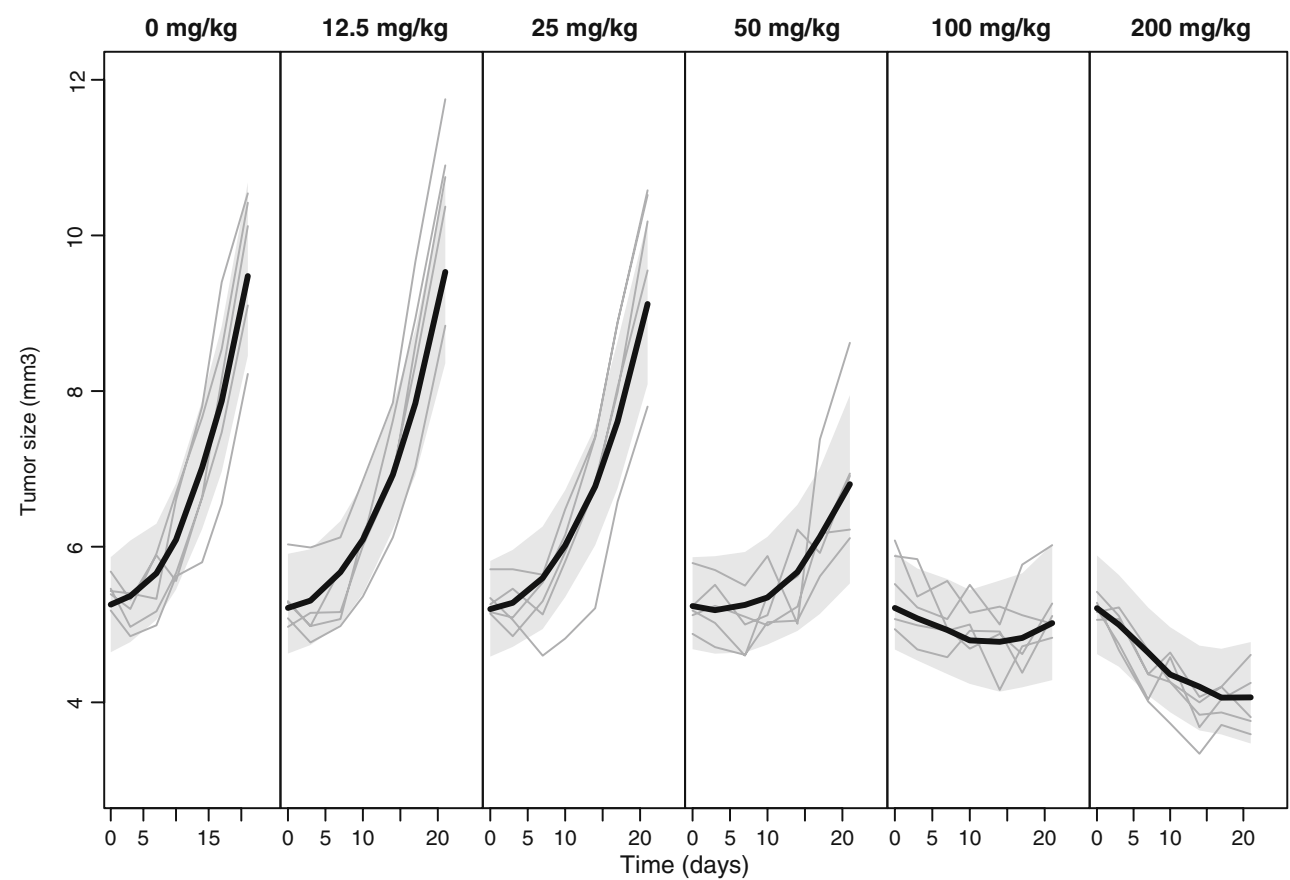

Fig. 2. Tumor growth curves in mice with implanted KP-1 tumors at different dose levels of E7820. The thin solid lines indicate observed growth curves, the black solid indicates model predicted growth curve, and the gray area indicate the $80 \%$ confidence interval of the model predictions

the mice with adequate precision for all dose levels, as can be seen in the plots in Fig. 2 which show both the observed tumor growth curves and the $80 \%$ of model predictions (i.e. between-mice variation in tumor size, and inhibition of tumor growth rates). The plots reveal that tumor growth was considerably inhibited from doses of $50 \mathrm{mg} / \mathrm{kg}$ upward, but that tumor stasis (relative to baseline) at $t=21$ was only achieved at doses of 100 and $200 \mathrm{mg} / \mathrm{kg}$. In Fig. 4, the relationships between dose level, inhibition of $\alpha_{2}$-integrin expression, and tumor growth inhibition are plotted, which were calculated from simulated tumor growth experiments. The inhibition of $\alpha_{2}$-integrin expression at steady state that is required for tumor stasis in $50 \%$ and $90 \%$ of mice $\left(I_{\text {inh,50 }}\right.$ and $I_{\text {inh,90 }}$ ) were calculated to be $14.7 \%$ (RSE 7\%)and $17.9 \%$ (RSE 8\%), respectively.

\section{Analysis of Clinical Trial Data}

In total, $462 \alpha_{2}$-integrin level measurements at 209 unique timepoints were available from 29 patients with solid tumors or lymphoma. The same turnover model that was used in the preclinical experiments was used to describe the $\alpha_{2}$-integrin profiles observed in the clinical trial. It was observed that the estimate for the turnover rate parameter $\left(k_{\text {out }}\right)$ in the clinical model $(0.099)$ was fairly similar to the estimate for the preclinical model (0.143), suggesting that rates of integrin inhibition are comparable, and only slightly faster in the preclinical setting. The estimate for $\mathrm{IC}_{50}$ value obtained for the clinical model was about fourfold higher than obtained for the preclinical model. Although BSV in baseline levels

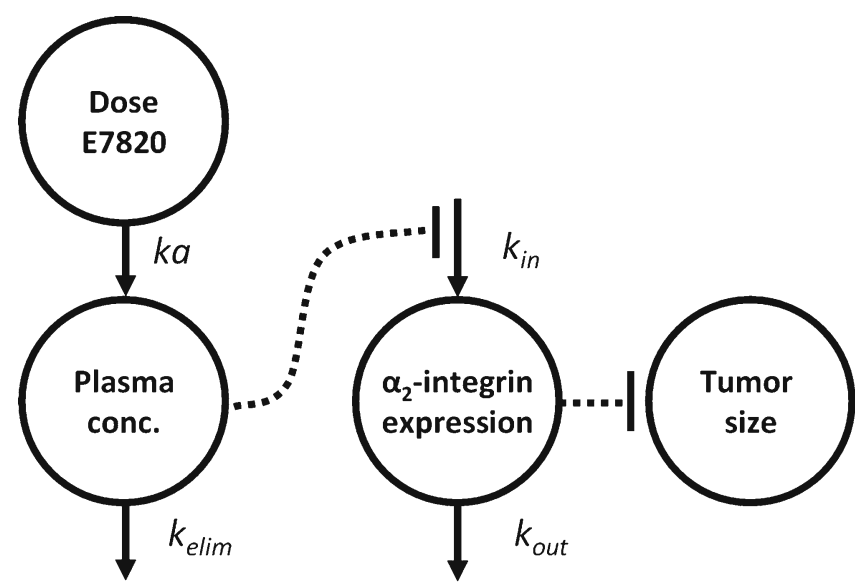

Fig. 3. Structure of the preclinical PK-PD-tumor growth model. The clinical model had the same structure, but did not incorporate a sub-model for tumor size 
Table II. PK Model Parameters Based on the Preclinical and Clinical Studies

\begin{tabular}{|c|c|c|c|c|}
\hline \multirow{2}{*}{$\frac{\text { Parameter }}{\mathrm{PK}}$} & & \multirow{2}{*}{$\frac{\text { Preclinical (mice) }}{\text { Estimates (IB) }}$} & \multicolumn{2}{|c|}{ Clinical } \\
\hline & & & Estimates(5) & RSE \\
\hline$C L^{a}$ & Clearance & $0.471 \mathrm{~L} \mathrm{~h}^{-1} \mathrm{~kg}^{-1}$ & $6.24 \mathrm{~L} \mathrm{~h}^{-1}$ & $5 \%$ \\
\hline$V^{a}$ & Volume of distribution & $0.684 \mathrm{~L} \mathrm{~kg}^{-1}$ & $66.0 \mathrm{~L}$ & $8 \%$ \\
\hline$F$ & Oral bioavailability & $69.3 \%$ & - & \\
\hline ka & Absorption rate & $3^{b} \mathrm{~h}^{-1}$ & $0.889 \mathrm{~h}^{-1}$ & $7 \%$ \\
\hline$\omega_{\mathrm{CL}}$ & $\mathrm{BSV}$ in clearance $(\mathrm{CV})$ & - & $51 \%$ & $12 \%$ \\
\hline$\omega_{V}$ & $\mathrm{BSV}$ in volume of distribution $(\mathrm{CV})$ & - & $48 \%$ & $17 \%$ \\
\hline$\rho_{\mathrm{CL} \sim V}$ & Correlation $C L \sim V(\mathrm{CV})$ & - & $62 \%$ & $12 \%$ \\
\hline
\end{tabular}

${ }^{a}$ Clinical estimates for clearance and volume of distribution are given as CL/F and V/F

${ }^{b}$ Absorption rate unknown, but assumed rapid $\left(3 \mathrm{~h}^{-1}\right)$ in preclinical experiments $\left(t_{\max }\right.$ was observed at $\left.\sim 1 \mathrm{~h}\right)$

$B S V$ between subject variation, $R S E$ Relative standard error (\%), $C V$ coefficient of variation (\%)

and drug sensitivity were high, significant effects of plasma drug exposure on the inhibition of $\alpha_{2}$-integrin expression were observed, and an $E_{\max }$ model best described this relationship. The data allowed the estimation of BSV in baseline $\alpha_{2}$-integrin expression levels and drug sensitivity $\left(\mathrm{IC}_{50}\right)$. A delay in drug effect did not improve the model, nor did a model that incorporated development of tolerance to E7820. Figure 5 shows a visual predictive check of the model and the data, in which dose levels of less than or equal to $50 \mathrm{mg} \mathrm{qd}$, and dose levels of greater than or equal to $70 \mathrm{mg}$ were grouped. This figure shows that the observed profiles correspond to the median model predicted profiles, and that most of the observed profiles are contained within the $90 \%$ confidence interval of the model predictions.

\section{Simulations of Clinical Regimens}

In Fig. 6, the results of simulations for the four highest dose levels that were studied in the phase I trial are plotted, along with the steady state level of $\alpha_{2}$-integrin expression inhibition corresponding to tumor stasis in $50 \%$ and $90 \%$ of mice. In Table $\mathrm{V}$, the average inhibition of $\alpha_{2}$-integrin expression $\left(I_{\text {int,av }}\right)$ after one cycle of E7820 of 28 days in patients is shown. These results demonstrate that a regimen of $50 \mathrm{mg}$ qd is expected to result in less than $50 \%$ of patients achieving the defined target of both $I_{\text {inh,50 }}$ and $I_{\text {inh,90. At }}$ continuous dosing at $70 \mathrm{mg} \mathrm{qd}$, inhibition of $\alpha_{2}$-integrin expression is expected to achieve the $I_{\text {inh,50 }}$ target in $>50 \%$ of patients, but the median expected integrin level is slightly lower than the $I_{\mathrm{inh}, 90}$. At doses of $100 \mathrm{mg} \mathrm{qd}$, median inhibition is higher than both targets, although the $95 \%$ confidence interval was not entirely higher than the $I_{\text {inh, } 90}$ target. Only at the highest evaluated but toxic dose of $200 \mathrm{mg}$ qd are $95 \%$ of patients expected to achieve inhibition of expression higher than both targets. This dose level has however been shown to induce non-tolerable hematological toxicity (15).

\section{DISCUSSION}

We have presented here a first population PK-PD analysis of preclinical and clinical data of the $\alpha_{2}$-integrin inhibitor E7820, and evaluated relationships between exposure to E7820, $\alpha_{2}$-integrin expression and tumor growth. Integrin inhibitors have been introduced recently as potential new treatment modalities in anti-cancer treatment that inhibit tumor growth by inhibiting angiogenesis. Integrins are heterodimer transmembrane receptors that are essential factors in angiogenesis as they mediate endothelial cell migration, proliferation and survival (16). Preclinical studies have shown that the inhibition of integrins, either by

Table III. PD Model Parameters for Integrin Model Based on the Preclinical and Clinical Studies

\begin{tabular}{|c|c|c|c|c|c|}
\hline \multirow{2}{*}{\multicolumn{2}{|c|}{$\begin{array}{l}\text { Parameter } \\
\text { PD: } \alpha_{2} \text {-integrin model }\end{array}$}} & \multicolumn{2}{|c|}{ Preclinical (mice) } & \multicolumn{2}{|c|}{ Clinical } \\
\hline & & \multirow{2}{*}{$\begin{array}{c}\text { Estimates } \\
22.5 \%\end{array}$} & \multirow{2}{*}{$\begin{array}{l}\text { RSE } \\
(1 \%)\end{array}$} & \multirow{2}{*}{$\begin{array}{c}\text { Estimates } \\
8,350 \mathrm{MESF}\end{array}$} & \multirow{2}{*}{$\begin{array}{l}\mathrm{RSE} \\
1 \%\end{array}$} \\
\hline$I_{\text {base }}$ & Baseline integrin level & & & & \\
\hline$K_{\text {in }}$ & Input rate turnover model & $3.21 \%$ day $^{-1}$ & $(19 \%)$ & 825.6 $\mathrm{MESF}$ day $^{-1}$ & $14 \%$ \\
\hline$K_{\text {out }}^{b}$ & Output rate turnover model & 0.143 day $^{-1}$ & & 0.099 day $^{-1}$ & \\
\hline$E_{\max , C}$ & $\begin{array}{l}\text { Maximal effect of E7820 } \\
\text { exposure on integrin inhibition }\end{array}$ & $1^{a}$ & & $1^{a}$ & \\
\hline $\mathrm{IC}_{50}$ & $\begin{array}{l}\text { Concentration at } 50 \% \text { of maximal } \\
\text { effect }\end{array}$ & $656 \mathrm{ng} \mathrm{ml}^{-1}$ & $(18 \%)$ & $2,840 \mathrm{ng} \mathrm{ml}^{-1}$ & $32 \%$ \\
\hline$\omega_{I, \text { base }}$ & $\mathrm{BSV}$ in integrin baseline & $31 \%$ & $(40 \%)$ & $40.0 \%$ & $19 \%$ \\
\hline$\omega_{\text {eff }}$ & BSV in sensitivity to E7820 & - & - & $110 \%$ & $32 \%$ \\
\hline$\sigma_{\exp }$ & Exponential residual error $(\mathrm{CV})$ & $6.7 \%$ & $(8 \%)$ & $11.5 \%$ & $23 \%$ \\
\hline
\end{tabular}

$M E S F$ molecules of equivalent soluble fluorochrome

${ }^{a}$ Fixed

${ }^{b}$ Not estimated, but calculated as $I_{\text {base }} / K_{\text {in }}$ 
Table IV. PD Model Parameters for the Tumor Growth Model Based on the Preclinical and Clinical Studies

\begin{tabular}{|c|c|c|c|}
\hline \multirow{2}{*}{\multicolumn{2}{|c|}{$\frac{\text { Parameter }}{\text { PD: Tumor growth model }}$}} & \multicolumn{2}{|c|}{ Preclinical (mice) } \\
\hline & & \multirow{2}{*}{$\begin{array}{l}\text { Estimates } \\
5.14 \mathrm{~mm}\end{array}$} & \multirow{2}{*}{$\begin{array}{c}\text { RSE } \\
1 \%\end{array}$} \\
\hline$T_{\text {base }}$ & Baseline tumor size & & \\
\hline$\alpha$ & Maximal exponential growth rate & 0.0903 day $^{-1}$ & $103 \%$ \\
\hline$\beta$ & Rate of initial resistance to tumor growth & 0.0391 day $^{-1}$ & $130 \%$ \\
\hline$E_{\mathrm{max}, I}$ & Maximal effect of integrin inhibition on tumor growth & 0.0472 day $^{-1}$ & $5 \%$ \\
\hline $\mathrm{II}_{50}$ & Relative inhibition of integrin expression at $50 \%$ of maximal effect & $11.4 \%$ & $6 \%$ \\
\hline$\omega_{T, \text { base }}$ & BSV in baseline tumor size & $6 \%$ & $13 \%$ \\
\hline$\omega_{\text {II50 }}$ & BSV in sensitivity to integrin expression inhibition & $20 \%$ & $50 \%$ \\
\hline$\sigma_{\exp }$ & Exponential residual error $(\mathrm{CV})$ & $6 \%$ & $6 \%$ \\
\hline
\end{tabular}

monoclonal antibodies, peptide drugs, or small molecules can result in tumor remission $(16,17)$. Currently, integrin inhibitors are investigational drugs and are in clinical and preclinical testing as single agents or in combination with other anti-cancer agents.

From experiments in mice, we were able to model with good accuracy the effects of drug exposure on levels of $\alpha_{2^{-}}$ integrin inhibition and tumor growth suppression in mice. The inhibition of $\alpha_{2}$-integrin expression levels was described by an indirect-effect model, which is the most widely used pharmacodynamic model for describing physiological processes. The model was able to describe the decrease in $\alpha_{2^{-}}$ integrin expression over time in response to treatment with E7820 and account for the between-mice variation in drug response. A simple exponential tumor growth model was then used to describe tumor growth profiles in mice; with the predicted $\alpha_{2}$-integrin expression levels driving growth inhibition A Gompertz model (13) provided worse fit than the empirical exponential model. Although tumor growth models have been presented in literature that is somewhat more physiologically based than the exponential model $(14,18)$, this model could also not describe our tumor growth better than the exponential model. More importantly, as the aim of this study was not to scale or extrapolate the tumor growth model to other species or other drugs, but primarily in accurately describing the relationship between $\alpha_{2}$-integrin expression and tumor growth, the exponential model was deemed most adequate. It should be stressed that the term $\left(1-e^{-\beta \cdot t}\right)$ included in the model does not describe drug resistance but rather initial slow tumor to growth, and that the rate parameter $\beta$ was estimated solely based on the unperturbed growth curves. The initial slow growth rate may be due to the transplanted tumor not being fully embedded yet in the tumor environment, and thus not fully able to receive nutrients and oxygen needed for growth. Although administration of E7820 started 7 days after transplantation of the tumor, this period may thus not be long enough to achieve the full potential tumor growth rate. It was noticed that $t_{1 / 2}$ was much lower in mice than in humans (1.01 h versus $7.33 \mathrm{~h})$. Additionally, relatively higher doses were administered to a

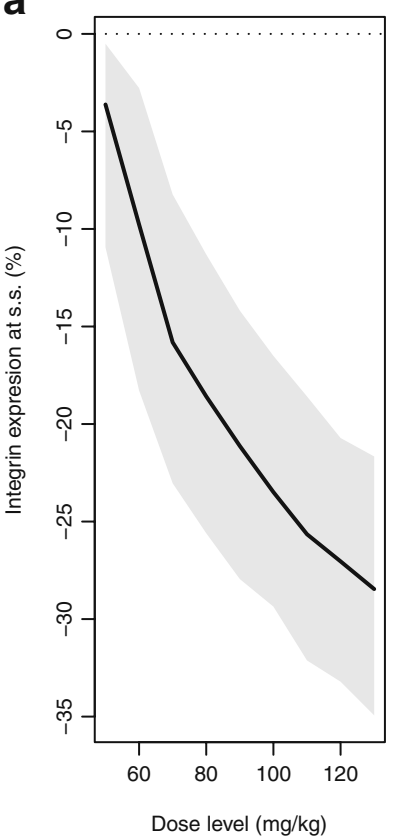

b

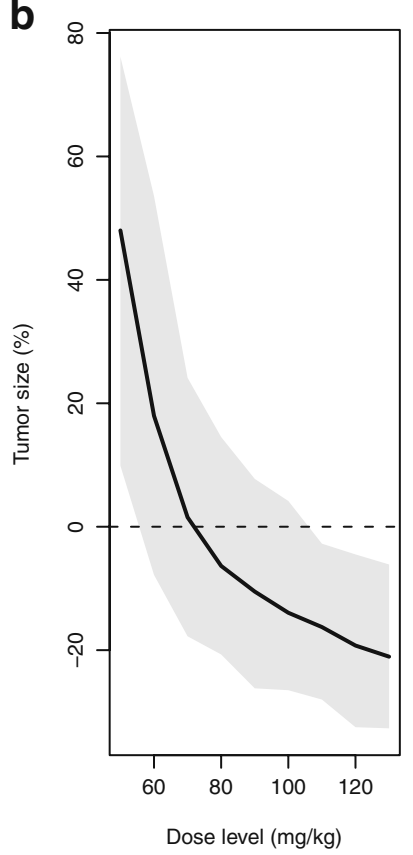

C

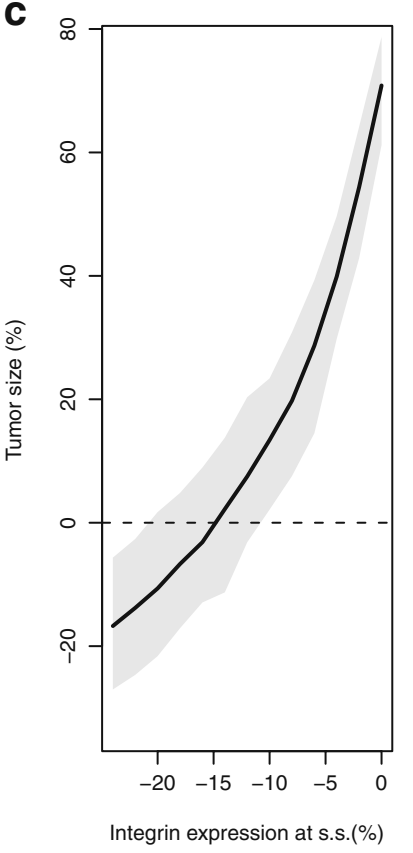

Fig. 4. a-c. Relationships between dose level, relative integrin inhibition at steady state, and relative tumor size measured in the tumor growth experiments in mice at $t=21$ days. Solid line indicates median, gray area indicates $90 \%$ prediction interval (variation between mice) 
a

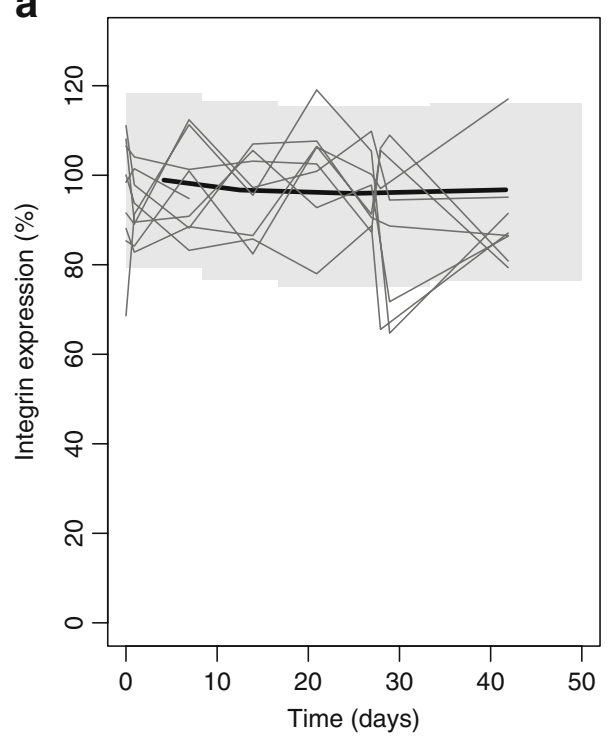

b

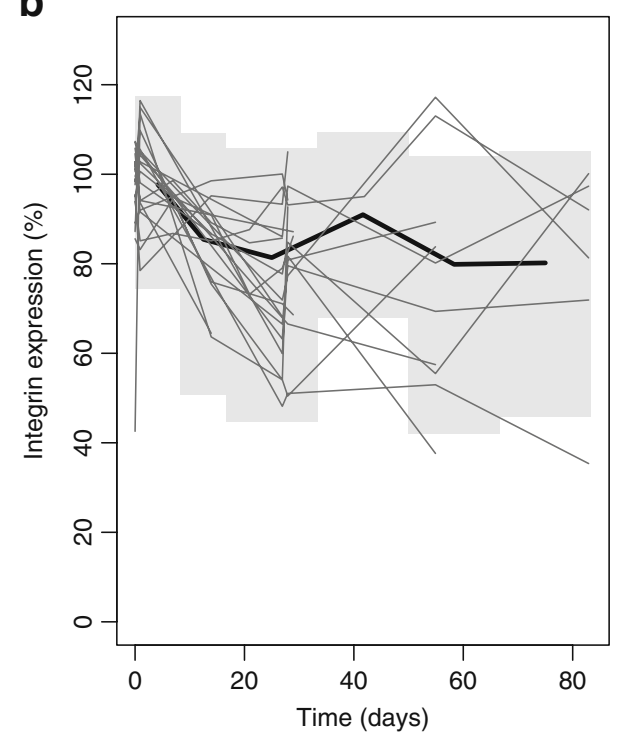

Fig. 5. a-b. Visual predictive check of the PK-PD model for $\alpha_{2}$-integrin expression on platelets in patients, for dose levels of $\leq 50 \mathrm{mg}$ qd (a), and $\geq 70 \mathrm{mg}$ qd (b). The thin solid lines show the individual observed integrin expression levels. Thick solid lines indicate model predicted median integrin expression over time, while the gray area indicates the $90 \%$ interval of the predicted between patient variation

mice than humans (maximum doses $200 \mathrm{mg} / \mathrm{kg}$ in mice $v s$ $200 \mathrm{mg}$ in humans). Therefore, much higher $C_{\max }$ concentrations were reached in mice, although E7820 was cleared faster. This may be important: apparently higher concentrations were required in mice compared to humans to elicit a response in integrin expression. However, inhibition of integrin expression in patients was already observed at the MTD of $100 \mathrm{mg}$, and therefore higher doses were not required to achieve pharmacological activity.
Using simulations from the combined preclinical model for E7820 exposure, $\alpha_{2}$-integrin and tumor growth, targets levels of $\alpha_{2}$-integrin inhibition that are expected to lead to tumor stasis in $50 \%$ and $90 \%$ of mice, $I_{\text {inh,50 }}$ and $I_{\text {inh,90, were }}$ estimated. The PK-PD model describing the relationship between E7820 exposure and $\alpha_{2}$-integrin expression in patients was then used to evaluate if these targets could be met with the regimens that were investigated in the clinical trial. This analysis showed that only at the highest toxic dose

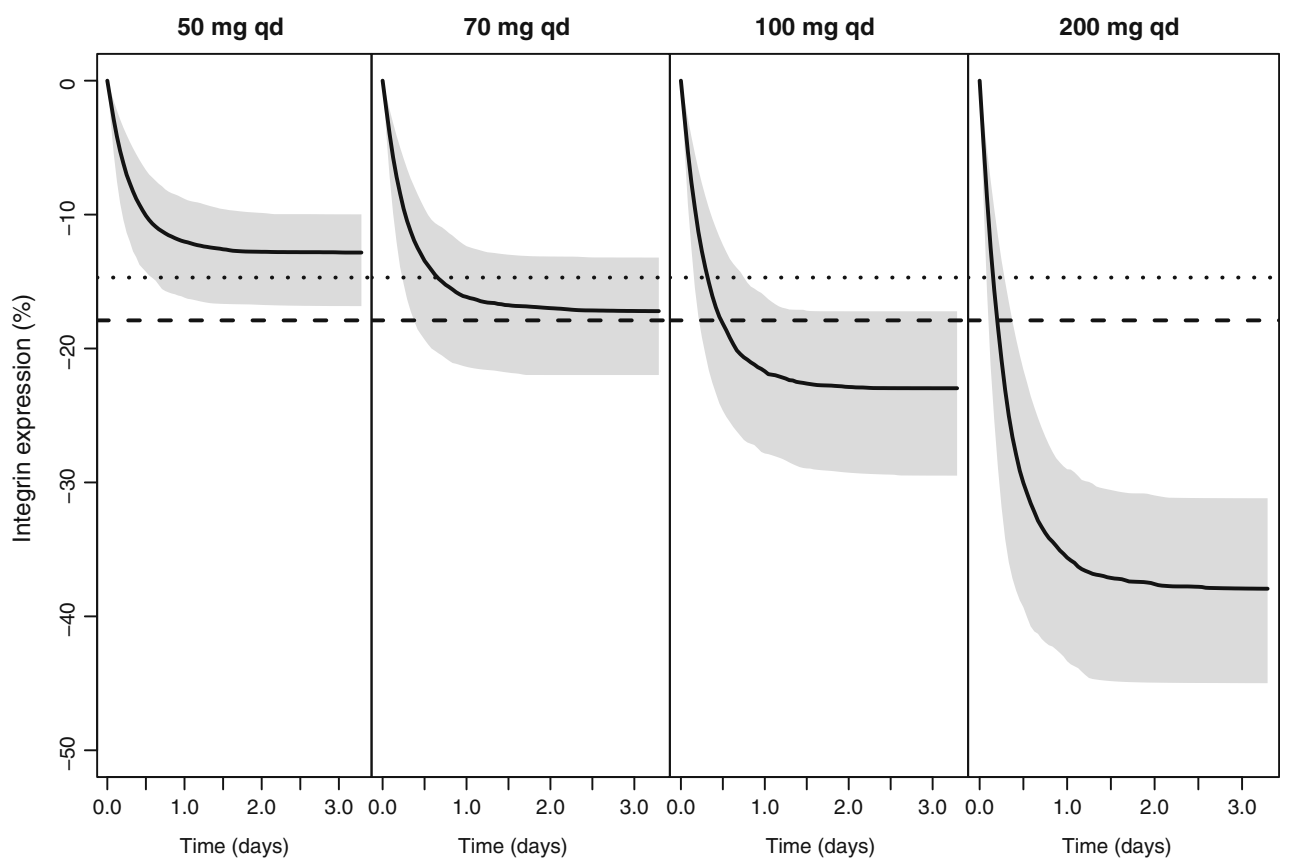

Fig. 6. Expected $\alpha_{2}$-integrin expression profiles (relative to baseline, without residual variability) during four continuous dosing regimens (qd). Solid lines indicate median expected $\alpha_{2}$-integrin expression levels, gray areas indicate the $90 \%$ model prediction intervals. Dotted and dashed lines indicate the $I_{\mathrm{inh}, 50}$, and $I_{\text {inh,90 }}$ (the average daily $\alpha_{2}$-integrin expression inhibition needed for $50 \%$ and $90 \%$ of mice to reach tumor stasis, respectively) 
Table V. Expected Relative Integrin Expression Inhibition $\left(I_{\text {int.av }}\right)$ at Steady State, Obtained from Simulations of Clinical Dosing Regimens

\begin{tabular}{cc}
\hline Dosing regimen & Expected $I_{\text {int,av }}$ at $t=21(\mathrm{CI} 95 \%)$ \\
\hline $50 \mathrm{mg} \mathrm{qd}$ & $12.8 \%(9.1 \%-17.7 \%)$ \\
$70 \mathrm{mg}$ qd & $17.2 \%(11.9 \%-23.3 \%)$ \\
$100 \mathrm{mg} \mathrm{qd}$ & $23.0 \%(16.5 \%-30.2 \%)$ \\
$200 \mathrm{mg} \mathrm{qd}$ & $37.8 \%(29.1 \%-48.0 \%)$ \\
\hline
\end{tabular}

of $200 \mathrm{mg} \mathrm{qd}$, both targets were met in more than $95 \%$ of patients. However, a continuous daily dose of $100 \mathrm{mg}$ would achieve the lower target $\left(I_{\text {inh,50 }}\right)$ in more than $95 \%$ of patients, and more than $50 \%$ of patients would achieve the defined high target. These results suggest that, for tumors that are susceptible to reduced $\alpha_{2}$-integrin expression, the proposed dose of $100 \mathrm{mg}$ qd is likely to achieve sufficient inhibition of $\alpha_{2}$-integrin expression to achieve tumor growth inhibition. Based on this analysis, doses of $50 \mathrm{mg}$ qd or lower are however unlikely to achieve efficacy.

This analysis provides evidence to support the notion that relative $\alpha_{2}$-integrin expression as measured on platelets may serve as a clinical biomarker for treatment efficacy. Considerable variation in baseline $\alpha_{2}$-integrin expression was observed between patients, thereby diminishing the predictive performance of absolute expression levels. However, limited experiments in healthy volunteers have shown that the expression is constant over time (1) and analysis of the data from the clinical study shows that expression remained constant in patients receiving low doses of E7820. A significant effect of drug exposure on the inhibition of $\alpha_{2}$-integrin expression was found. This suggests that the level of $\alpha_{2}$-integrin expression as measured on platelets relative to the baseline level could serve as a biomarker to assess target modulation in response to treatment with E7820. Since the $\alpha_{2}$-integrin inhibition targets obtained from the preclinical experiments were low $(<20 \%)$, this suggest that even moderate inhibition of $\alpha_{2}$-integrin expression on platelets is correlated with tumor growth inhibition.

In the development of new drugs, results from preclinical efficacy and toxicity experiments are generally only used to serve as input for the early clinical stage, i.e., to provide a safe starting dose. Once in the clinical stage, results from preclinical efficacy experiments, i.e., tumor growth experiments, are often not taken into account. In this analysis, in contrast, we present an integrated model-based analysis of all data currently available on E7820: data obtained from preclinical experiments were projected onto data obtained from a clinical trial. As data from the preclinical stage may hold valuable information on the in vivo drug response, especially when combined with data on a suitable pharmacodynamic biomarker, such an analysis may allow an early assessment of efficacy.

It is currently unknown how different tumor types will respond to E7820 exposure, and to the inhibition of integrin expression. It is also not clear yet which tumor types respond best to this compound. Likely, not all tumor types will be sensitive to the inhibition of integrin, and therefore the value of integrin expression as a biomarker must be assessed in more detail in subsequent studies. The preclinical PD model was built using data obtained using a pancreatic cell line. If more preclinical data would become available, the model could be updated with other cell-lines as well, to provide a more robust model, or a model tailored to specific tumor types. Another important consideration with this analysis is the role that integrins play in mouse compared to humans. In the preclinical experiments, integrins on platelets were of mouse origin, while those on tumor were of human origin. How this impacts the results is unclear, and calls for caution in interpretation of the results. Another consideration is that tumor growth rates and integrin turnover rates may differ between mice and humans. Additionally, as in patients, larger, necrotic, and more variably sized tumors are encountered and blood supply to tumors is likely to differ between the two species. Further clinical investigation is therefore needed to confirm the results of this analysis. Additional biomarker and tumor growth collected from a homogeneous group of patients will allow refinement of the current model, and will establish validity of $\alpha_{2}$-integrin as a biomarker for tumor growth inhibition. However, in our opinion, the analysis presented here is the most informative analysis that is possible at the current stage of development of this drug, as it not only takes into account the clinical results but also results from the preclinical phase.

Open Access This article is distributed under the terms of the Creative Commons Attribution Noncommercial License which permits any noncommercial use, distribution, and reproduction in any medium, provided the original author(s) and source are credited.

\section{REFERENCES}

1. Semba T, Funahashi Y, Ono N, et al. An angiogenesis inhibitor E7820 shows broad-spectrum tumor growth inhibition in a xenograft model: possible value of integrin alpha2 on platelets as a biological marker. Clin Cancer Res. 2004;10:1430-8.

2. Funahashi Y, Sugi NH, Semba T, et al. Sulfonamide derivative, E7820, is a unique angiogenesis inhibitor suppressing an expression of integrin alpha2 subunit on endothelium. Cancer Res. 2002;62:6116-23.

3. Senger DR, Perruzzi CA, Streit M, Koteliansky VE, de Fougerolles AR, Detmar M. The alpha(1)beta(1) and alpha(2)beta(1) integrins provide critical support for vascular endothelial growth factor signaling, endothelial cell migration, and tumor angiogenesis. Am J Pathol. 2002;160:195-204.

4. Mita MM, Mita AC, Goldston M, et al. Pharmacokinetics (PK) and pharmacodynamics (PD) of E7820-an oral sulfonamide with novel, alpha-2 integrin mediated antiangiogenic properties: results of a phase I study. J Clin Oncol (Meeting Abstracts). 2005;23:3082.

5. Keizer RJ, Zamacona M, Jansen M, et al. Application of population pharmacokinetic modeling in early clinical development of the anticancer agent E7820 [Internet]. Investigational New Drugs 2008; [cited 2008 Aug 25] Available from: http://www. ncbi.nlm.nih.gov/pubmed/18712503

6. Sawyer MB, Iqbal S, Lenz H, et al. Phase II study of E7820 in combination with cetuximab in subjects (pts) with metastatic and refractory colorectal cancer (CRC). J Clin Oncol (Meeting Abstracts). 2010;28:3537.

7. Tanaka C, O'Reilly T, Kovarik JM, et al. Identifying optimal biologic doses of everolimus (RAD001) in patients with cancer based on the modeling of preclinical and clinical pharmacokinetic and pharmacodynamic data. J Clin Oncol. 2008;26:1596-602. 
8. Wang S, Zhou Q, Gallo JM. Demonstration of the equivalent pharmacokinetic/pharmacodynamic dosing strategy in a multiple-dose study of gefitinib. Mol Cancer Ther. 2009;8:1438-47.

9. Piraña and PCluster: a modeling environment and cluster infrastructure for NONMEM. Keizer RJ, van Benten M, Beijnen JH, Schellens JH, Huitema AD. Comput Methods Programs Biomed. 2011;101(1):72-9.

10. Holford, N. VPC, the visual predictive check-superiority to standard diagnostic (Rorschach) plots. In: PAGE 2005. 2005.

11. Dayneka NL, Garg V, Jusko WJ. Comparison of four basic models of indirect pharmacodynamic responses. J Pharmacokinet Biopharm. 1993;21:457-78.

12. Dansirikul C, Silber H, Karlsson M. Approaches to handling pharmacodynamic baseline responses. J Pharmacokinet Pharmacodyn. 2008;35:269-83.

13. Iliadis A, Barbolosi D. Optimizing drug regimens in cancer chemotherapy by an efficacy-toxicity mathematical model. Comput Biomed Res. 2000;33:211-26.
14. Simeoni M, Magni P, Cammia C, et al. Predictive pharmacokinetic-pharmacodynamic modeling of tumor growth kinetics in xenograft models after administration of anticancer agents. Cancer Res. 2004;64:1094-101.

15. Mita MM, Mita AC, Ricart A, et al. Phase I study of an antiangiogenic agent with a novel mechanism of action E7820: Safety, pharmacokinetics (PK) and pharmacodynamic (PD) studies in patients (pts) with solid tumors. J Clin Oncol (Meeting Abstracts). 2006;24:3048.

16. Alghisi GC, Rüegg C. Vascular integrins in tumor angiogenesis: mediators and therapeutic targets. Endothelium. 2006;13:11335.

17. Jin H, Varner J. Integrins: roles in cancer development and as treatment targets. Br J Cancer. 2004;90:561-5.

18. Bueno L, de Alwis DP, Pitou C, et al. Semi-mechanistic modelling of the tumour growth inhibitory effects of LY2157299, a new type I receptor TGF-beta kinase antagonist, in mice. Eur J Cancer. 2008;44:142-50. 